\title{
USING THE NETWORKED KNOWLEDGE ACTIVITIES FRAMEWORK TO EXAMINE LEARNING ON SOCIAL NETWORKING SITES
}

\author{
Vanessa P. Dennen, Kari Word, Dawn Adolfson, Victor Arechavaleta, Dan He, \\ Chia-Wei Hsu, Jaesung Hur, Daeun Jung, Heather Kent, Annemarie Russell and Korinna Toth \\ Florida State University, Tallahassee, FL, USA
}

\begin{abstract}
This study uses the Networked Knowledge Activities (NKA) framework to examine social media archival data for evidence of learning-related actions across six major social networking sites (SNS). Findings show that while the NKAs are present on and fostered by the tools available on most SNS, most non-verbal indicators of NKAs provide insufficient reliable evidence of a networked knowledge activity. User intent, which is not detectable unless articulated in a message by a user, must be known to accurately use SNS archives to measure networked knowledge activities. We recommend combining this approach with user surveys or interviews within the studied knowledge network to improve accuracy and better understand social media knowledge activities.
\end{abstract}

\section{KEYWORDS}

Informal Learning, Knowledge Activities, Knowledge Networks, Learning, Sharing

\section{INTRODUCTION}

Learning is one of many functions that can be supported via interactions on social networking sites. Although popular social networking sites (SNS) like Facebook and Twitter were not originally designed as learning tools, they have quickly been drafted into service in a variety of learning contexts. For example, Facebook groups have been used as an alternative to learning management systems for university classes (Meishar-Tal, Kurtz et al. 2012, Wang, Woo et al. 2012) as well as to support informal learning (Adalberon and Säljö 2017) and provide a space where students might blend social interaction and schoolwork (Aaen and Dalsgaard 2016). YouTube is a frequent content provider for students when they study (Moghavvemi, Sulaiman et al. 2018), but also hosts communities such as beauty bloggers through which informal learning is supported (Choi and Behm-Morawitz 2017). Twitter can swiftly unite groups of people with shared interests through hashtags to engage in professional development discourse (Greenhalgh and Koehler 2017). Although SNS are frequently discussed as relationship (Chambers 2013) and political spaces (Bond and Messing 2015), their function as learning spaces that support knowledge networks is equally robust even if less frequently considered.

In this study, we examine how different SNS support knowledge networks through their various features and tools, and consider how readily one can find evidence of these networked knowledge activities through an analysis of archived user actions and interactions. This knowledge has implications for educators seeking ways of assessing social media learning processes as well as researchers seeking ways of making meaning from social media archival data.

\section{NETWORKED KNOWLEDGE ACTIVITIES FRAMEWORK}

The Networked Knowledge Activities (NKA) Framework (Dennen 2019) articulates and describes the six discrete knowledge activities that people use in networked online contexts (e.g., online classes, social media, virtual communities of practice). These activities were identified through an examination of the complex 
ways that people naturally interact with knowledge objects and members of their online networks in the service of learning.

The six networked knowledge activities are summarized in Table 1. Although each can be uniquely described, the NKAs tend to not occur in isolation. In other words, they are likely to co-occur in the midst of an individual's learning process, or to flow from one to another. For example, collecting can lead to curating, and curated collections may be shared with one's network. Or, in response to a query within one's network, a resource might be brokered between networks, resulting in a discussion where meaning is negotiated. Should multiple network members offer resources, a collection may be formed.

The NKAs support three main knowledge functions: management, communication, and creation (Dennen 2019). Collecting and curating are both forms of knowledge management, whereas sharing, brokering, and negotiating are acts of communication. Curating and constructing both are forms of creation. When looking at this broader level, there are parallels to the $4 \mathrm{C}$ framework (Milligan, Littlejohn et al. 2014), which focuses on consuming, creating, connecting, and contributing. However, the NKA do not account for idle consumption, instead focusing on more purposeful knowledge management activities which are an extension of consumption. Additionally, the NKA framework groups the share, broker and negotiate activities as communication functions, whereas the $4 \mathrm{C}$ framework splits these into two areas, connect and contribute. Essentially, the NKA framework pulls out negotiate (the discursive process of collaboratively exploring ideas and making meaning) as an activity that stands alone.

Table 1. Overview of networked knowledge activities

\begin{tabular}{|c|c|}
\hline Activity & Description \\
\hline Collect & To save a copy of or link to an item so it can be found or retrieved at a later time. \\
\hline Curate & $\begin{array}{l}\text { Creating organized, annotated collections of online artifacts. More purposeful and selective than } \\
\text { collecting, and often follows the collection process. }\end{array}$ \\
\hline Share & To take something has been collected, curated, or constructed and make it available online. \\
\hline Broker & $\begin{array}{l}\text { The act of connecting online and offline groups or networks via knowledge transmission. The } \\
\text { broker acts as a purposeful intermediary agent between groups. }\end{array}$ \\
\hline Negotiate & A collaborative, discursive process in which learners work together to agree upon meaning. \\
\hline Construct & $\begin{array}{l}\text { The act of making something new or combining existing things in a new manner. Often an } \\
\text { outcome of knowledge negotiation. Results in a product that can be shared with others. }\end{array}$ \\
\hline
\end{tabular}

The NKA framework's initial purpose was to guide the design and development of social media-based learning activities in formal learning contexts. To that end, the different activities could be used in conjunction with Bloom's Taxonomy (Bloom 1956, Krathwohl and Anderson 2009) or Gagne's domains of learning (Gagne 1984) to help instructors design lessons supported by social networking sites and related online tools. By aligning activities with desired learning outcomes, learners can be guided toward purposeful online interactions.

While this framework is useful for supporting instructional design processes, its ability to guide assessment and measurement has previously been unexamined. Of particular interest is whether there are visible indicators of NKAs in social media archives, and what these indicators might be. The ability to easy identify NKAs in archival data could be helpful for instructors who are assessing student work, especially work processes. It would also be useful for researchers seeking evidence of knowledge and learning activities among different online networks and spaces. 


\section{METHODS}

The research questions that guided this study are:

1. Which networked knowledge activities are readily visible through user actions on popular SNS?

2. What user actions on SNS indicate different networked knowledge activities?

3. How do the tool features and norms enable or prohibit the ability to perform and identify NKA?

4. How do NKA flow across or connect different SNS?

To investigate our research questions, we reviewed authentic user posts and interactions in spaces and contexts where people were known to be engaged in classroom, informal, and professional learning.

We focused on six SNS (Facebook, Instagram, Twitter, Pinterest, LinkedIn, YouTube). These were selected based on their broad popularity with teens (Anderson and Jiang 2018) and adults (Anderson and Perrin 2017, Perrin and Anderson 2019), and for their prior research connections to classroom learning (Salomon 2013, Lapolla 2014), informal learning (Greenhow and Lewin 2016, Bagdy, Dennen et al. 2018, Brown Jarreau, Dahmen et al. 2019), or professional development (Carpenter and Krutka 2015, Hu, Torphy et al. 2018, Bridgstock 2019) activities. Our primary data sources were groups and hashtags where informal learning and professional development activities occurred. We did not focus on formal learning contexts because an instructor-led context is a bit different, and instructors may provide specific guidance related to how they want students to share, tag, like, and comment. Most data sources involved English language messages, but our multi-linguistic research team also incorporated data sources posted in Chinese and Korean.

Sub-groups of 2-3 researchers on the larger research team chose individual SNS to focus on. In these sub-groups, the researchers looked across interactions in different group and network contexts to find evidence of different NKAs. Contextual cues in comments were relied on to confirm non-verbal NKA evidence, noting that in many instances of the same NKA the corresponding comments may be absent. When evidence was found, they considered whether the same evidence would be present in each instance of the NKA, or if it was user dependent. For example, one indicator of brokering might be to state in a message something similar to "This item came from this other group to which I belong, and I am sharing it here because it relates to the question you are asking." However, a person could broker knowledge in this way without formally acknowledging the act, making it difficult to distinguish sharing from its more purposeful counterpart, brokering. When clear or unambiguous evidence of a particular NKA was not found, the researchers considered whether the NKA was likely supported by that SNS, and the various means through which the NKA might occur. Throughout this investigation, the manner in which specific tool features facilitated NKAs was documented, and group members tested features to see how well they would support different NKAs. Once each sub-group completed their individual investigation of NKAs on a specific SNS, they shared their findings with the larger research group. Through whole group discussion, these findings were refined, and comparisons made across the SNS.

\section{RESULTS}

\subsection{Facebook}

Collection is difficult to see on Facebook, although users may save or bookmark individual posts. Some users may use the emoji function to like a post, or post a comment (e.g., "F" or "follow") to ensure they can find and later review or collect the responses to that post. However, these are not reliable indicators; there are many reasons why a person may like a post or want to follow a post. Similarly, curation is difficult to see unless a user deliberately curates and shares resources within a post or across multiple posts. Alternately, a photo album may be evidence of curated visual materials on Facebook.

Sharing and brokering are facilitated via the sharing tools and the ability to tag other individuals and call their attention to a particular item. Both may also be invisible acts, with post shared from public pages to private messages or within closed or secret groups. Share counts show how many times the share function has been used, but tracking the locations of shares is difficult given varying privacy settings.

Facebook has a high degree of posting and commenting, with knowledge negotiation frequently visible in the comment-based dialogues. Again, privacy settings and shifting dialogues to messenger can obscure access to knowledge negotiation. Additionally, high rates of posting and commenting are not necessarily 
indicators of knowledge negotiation. It is necessary to examine the content of comments in order to make that determination.

Finally, knowledge construction may occur directly on Facebook through posts of varying types. Co-constructed knowledge often occurs in Facebook groups as an outgrowth of sharing and negotiation, or a focused curation effort. Additionally, media constructed in other tools are frequently shared on Facebook. Facebook is designed to support the flow of information in from other platforms. While this is useful for sharing purposes, the way that shared items on pages, timelines, and groups function make it poorly suited for collecting and then curating knowledge objects from other platforms.

\subsection{Twitter}

Much like on Facebook, it is difficult to see collection on Twitter, although users can save and return to tweets through three Twitter-based functions, favorites, bookmarks, and retweets. Each of these actions ties the original tweet back to the collector's Twitter account. However, it is difficult to say with certainty when favorites and retweets are used in this manner versus for another purpose. Twitter makes analytic data about tweets available, but like and retweet counts, while indicators of message visibility and amplification, do not reliably demonstrate collection. Bookmarks are only visible to the individual who did the bookmarking. Similarly, curation has limited visibility on Twitter. The moments feature, which was infrequently seen, can help connect a set of tweets in a curation-like manner. What remains unknown is how many people click through content on Twitter and then proceed to collect or curate the shared content on another platform.

Sharing is at the heart of Twitter use in a networked knowledge context. Sharing does not mean hosting knowledge on Twitter, but rather pointing to knowledge objects that are hosted elsewhere. Sharing may co-occur with negotiation when a person shares a knowledge item and others proceed to comment on and discuss it. occurs on Twitter in the form of an initial tweet with the intended purpose of sharing knowledge. Twitter shares that originate on another site (e.g., sharing from a news source) tend to carry over not only a link to shared content but an@mention that tags the original source on Twitter. People who share items can also add their own@mentions to draw a specific person's attention to a tweet, which would them be demonstrative of knowledge brokering. Although sharing is a high frequency activity on Twitter, deciphering the intent of a share is complex without clear verbal indicators. Additionally sharing, brokering, and negotiation may occur that is not visible when private messaging is used or tweets are linked or embedded and potentially discussed on other platforms.

When knowledge negotiation occurs on Twitter, it takes the form of a conversation among individuals responding to each other. The @ reply function helps continuously tag people into the conversation and organize it in threads. Of course, not every reply is an act of negotiation. Some are purely social, entertaining, or may be offered as acknowledgements or answers to brief questions. It is necessary to view the content of a twitter thread to know whether knowledge negotiation actually took place.

Hashtags also can be used to support multiple knowledge activities on Twitter. Hashtags serve as aggregators, and can help unite a larger network in discussion, as in the case of Twitter chats; share a resource with likeminded others; or mark and collect tweets in a personally meaningful way. However, hashtags have also been used as performative expressions of attitude (e.g., \#dontcare), and may not be meant to aggregate tweets topically or by network, or to support knowledge activities.

Finally, although some people regularly use Twitter as a means of sharing the pictures, videos, blogs, and other content they have created outside of Twitter, it is not a platform where that construction is likely to occur. This is probably due to Twitter's character and content hosting limitations. That said, there have been instances where Twitter has been used as a form of writing, and a novel artefact has been constructed primarily on Twitter. While intriguing, this is not how most people use Twitter in their knowledge networks.

\subsection{Instagram}

Instagram is a heavily visual platform, with supporting tools that facilitate liking, commenting, sharing, and tagging. On Instagram, similar to the other platforms, the bookmark and like functions potentially support collection activities. Through bookmarking, images posted by others can be stored in personal "collection" that is not shared with others. The like button, triggered by clicking on a heart, has been a controversial features and the platform is experimenting with its removal of public likes (Al-Heeti 2019), which will likely have minimal effect on networked knowledge activities. Liking is not a useful means of marking Instagram posts for collection because the interface makes it difficult to compile liked items. 
An Instagram user can also curate information from their own collections. After bookmarking posts, a user can make new folders into which the posts can be saved and organized. Individual posts can be hidden from a user's feed as well, to ensure that the feed itself has a curated look and feel. Feed curation is an important activity for many Instagram users. Instagram also has a story feature that supports temporary sharing of images. After a day, story posts are no longer visible unless they have been otherwise saved.

As seen on other SNS, Instagram supports hashtags which can be used to aggregate posts on a topic. This feature may be used to support curation activities when users consistently tag images they wish to search and access together or share to others as an intentionally grouped collection. It also can support a collaborative form of collection. For example, co-learners or events may use a hashtag to pool together their images. Hashtags also may be used to direct a specific post toward an affinity group, whether that group contains people who are within the individual's network or are simply people who are likely to search that hashtag.

Users share information on Instagram through both posts and comments. Instagram posts may videos, photos, or other images. Text may be incorporated directly onto a shared photo or image, or added as a caption to an image. or image with text information. Images can be constructed (i.e., captured and edited) directly on the SNS, or items constructed using other tools can be uploaded. Many third-party tools have been developed to facilitate the construction of attractive graphics, including text, that meet the image dimensions on Instagram.

Unlike other platforms, Instagram does not have a dedicated function for sharing another user's posts, although these posts can be added to one's Instagram story. Third-party apps like can be used to integrate this functionality, called regramming, as desired. Additionally, although Instagram has a commenting feature, comments are not commonly used to support the level of discourse necessary to see knowledge negotiation. Perhaps this is because Instagram is typically associated with visuals, which are the centerpiece of every post, and the app space that is devoted to comments is small.

On the surface, Instagram may appear to have similarities to Facebook and Twitter, and when a user creates Instagram posts they can readily share those posts to their accounts on the other platforms. However, Instagram appears to foster NKAs in a much more limited manner than Facebook and Twitter. The sharing and negotiation tools are far less robust. Individuals may be searching Instagram for visual content to be used for knowledge purposes, but SNS like Pinterest, better serve the interests of people looking to collect, curate, and share visual knowledge.

\subsection{LinkedIn}

LinkedIn is a social networking site primarily used by professionals and organizations. In addition to hosting online profiles of one's work experiences, LinkedIn allows users to post content in the form of text, images, and videos. Each post allows users to react (e.g., like) or share. Not all NKAs may be visible on LinkedIn, but they are all likely to occur on LinkedIn.

Collection, which may lead to curation, occurs on LinkedIn through the "save links for later" option. Items saved for later are privately saved, which means that collection is not visible to third parties. One clue to items of interest are the reactions feature that offers five visual comments that can be quickly applied to posts. LinkedIn provides a resource to indicate their intended meaning although that does not guarantee their use in this manner. The two that seem most closely related to networked knowledge activities, the insightful and curious reactions, were rarely applied to posts. In contrast, like, love, and celebrate were heavily used.

Through posts, LinkedIn users can share their own written content and other knowledge objects (e.g., images, videos, slides, PDFs). Much of the knowledge objects that are shared are constructed outside of LinkedIn, which only supports the construction of articles within the platform. It is likely due to LinkedIn's focus on professional interactions that posts explicitly share knowledge in an informal or professional development context. Points of sharing occasionally turn into negotiation via conversation within comments. It should be noted that LinkedIn also contains many posts of a professional-social or broadcast announcement nature, such as statements about recent accomplishments, job transitions, or events and opportunities.

\subsection{Pinterest}

Pinterest supports users as they search, collect, and organize interesting knowledge objects on the internet. These objects can be images, websites, videos, documents, and more, and are represented on Pinterest with images that link through to more extensive content. User can take the knowledge objects that they have found and pin them to boards organized around specific topics. Boards can be constructed individually or 
collaboratively. then post, or pin, these items to a board that's organized around a specific topic. Similarly, boards can be searched and followed. Users can add other people to their networks to receive alerts about new pins that have been added.

The Pinterest platform is clearly focused on four of the NKAs: collect, curate, share, and construct. The collecting is done by saving pins onto boards. Boards can be general, open collections, or they may contain intentionally curated ones, with pinned items annotated by the person who pinned them. Pin collections may be kept private, or they can be shared with others. Pins frequently are shared to other sites, such as Facebook and Twitter. Additionally, many bloggers will curate and share pins on their blogs or websites. Pinterest also has a feature that allows users to email or text pins or boards to other users.

Finally, users can construct their own pins to share on Pinterest. Pins are created by using a Pinterest share link on another website or by posting the site URL on Pinterest to create a new pin. Pinterest will ask the user to select a display image for the pin and then the user can also annotate the pin and post it to one of their boards. If the pin was created publicly, then other users can re-pin that object to their own boards.

Some activities are not visible on Pinterest but could be happening, such as brokering. Pinterest's sharing tools do not provide clear indicators of sharing pathways and purposefulness. However, much of the outward flow of sharing, which may occur at the pin or board level, may be related to brokering activities. Negotiating is also unlikely to occur on Pinterest. Pinterest supports commenting, but this feature is infrequently used and likely reflects the network-dependent but still fairly solitary nature of collecting and curating items.

\subsection{YouTube}

YouTube hosts a vast expanse of videos, and the relative amount of NKA occurring on the platform is low. People can collect videos on YouTube by subscribing to them, saving playlists, liking videos, or downloading them. These acts allow an individual user to easily find their favorite videos. Curation occurs as users generate playlists, often facilitated by the YouTube recommender algorithm. Sharing and brokering videos are both facilitated through various means. A creator can upload and share to YouTube, and a consumer can use the built-in sharing tools to alert others to specific videos. Sharing can occur in video description fields, and in the comments, although in practice there relatively little discussion occurs in comments unless a channel community has been developed (e.g., popular youtubers). Finally, construction is possible through embedded video creation tools.

Playlists are a feature unique to YouTube, and merit discussion on their own. The playlist feature enables users to collect videos and revisit them. Within a playlist, a user can give a name to it, add any description, and reorder the videos to be played. A playlist can be set to be public, private, or unlisted (i.e., only people who know the direct link can view). Users can save videos to their YouTube library.

The playlist feature may lead to a curation if users intentionally make new playlists and organize videos for certain topics or purposes. For example, someone who is trying to study statistics can collect statistics lecture videos and make a new playlist, ordering the videos from basics to advanced. This playlist can be made public and shared so it benefits others. Furthermore, the playlist feature can be used for knowledge construction. For example, a history teacher who wants to teach students about a particular era can assemble video sources that share news, movies, songs, and other key details about the era, creating a comprehensive learning resource.

On YouTube the flow of video media onto the platform occurs via a user upload. Several video recording and editing tools have embedded sharing tools that connect direction to YouTube, facilitating the process of sharing one's personal creations. In a SNS NKA context, YouTube most often serves as a hosting platform for videos that are embedded into web pages and other platforms. In other words, there is a high degree of content flowing out of YouTube to support NKA.

Despite these findings that YouTube can support the NKA, many users continue to use YouTube in a passive sense, making their intent and potential use of NKA largely invisible. Video play counts greatly outnumber comments, saves, and appearances on playlists. Likes and dislikes are not clearly related to any knowledge activities. The play counts and likes/dislikes make clear that many users are present, but they do not appear to be heavily interacting with each other or building networks. 


\section{DISCUSSION AND CONCLUSION}

The NKA framework was useful to identify different types of knowledge activities that occur on SNS and separate them out from other SNS functions like social conversation, identity performance, and message broadcasting. At the same time, the NKA framework alone applied to a social media archive, while useful for identifying general user knowledge actions, may not be sufficient to fully identify and count all of these actions accurately. It is easiest to identify the presence of NKAs when people state their purpose (e.g., 'Thank you! I am saving this to review later.' or 'Another group I belong to created this resource and I think you might find it useful.'). However, it can be difficult to interpret user intent when tools such as like buttons, share buttons, and tags are used. For example, collect and curate use the same tools (bookmarks, downloads, likes), but with different ends; individuals who curate are more purposeful in their selection and organization processes.

Across all six SNS, collecting and curating tended to be individual activities, often done without leaving a meaningful indicator in archival data sets. Collaborative efforts to collect or curate were evident when networks shared group spaces or hashtags for the purpose of building knowledge bases. The norms that a network upholds for saving and organizing items determines whether the product would be considered a simple collection or a curated collection.

Sharing and brokering involve multiple participants, and can be directed at specific people or groups via tags and comments, but unless a visible acknowledgement of receipt is provided (e.g., a like, download, or comment) it is difficult to know if they have had any more effect on networked knowledge than shouting into an empty room. In contrast, negotiation inherently involves two or more active participants. With that noted, we did see instances in which people shared items while writing commentary that appeared to be an act of knowledge negotiation with the original author of the shared item. Although the author was unlikely to be tagged, and many of these shared items we saw were formally published articles and news stories, there is potential for tagging in the author of a knowledge object to try to engage them in further discourse.

Several SNS tools enable users to collect and share. Likes, favorites, and other forms of paralinguistic digital affordances (PDA) are perhaps the most interesting to explore, because the simple action of clicking a button can have multiple meanings (Hayes, Carr et al. 2016), especially in a knowledge activity context. It is less likely in this context that individuals use PDAs to provide social support as they do in other contexts (Wohn, Carr et al. 2016), although supporting network relationships and social capital may still be a concern (Fish 2018). Distinguishing among users who click on these indicators to say "I saw this," "I like this," and "I want to save this so I can find it later," can be difficult if there are not corresponding linguistic cues in a comment that explain the user's intent. On SNS like Twitter, likes can be easily revisited and reviewed. Although there is also a bookmark feature, it can be simpler to click a like to mark a tweet while scrolling past. Alternately, these indicators can be used as a shortcut way of saying "I saw this item." From there it is difficult to interpret whether the indicator is a marker of acknowledgement, agreement, or something else altogether. On SNS platforms with multiple PDA indicators (e.g., Facebook), interpreting a PDA click remains complicated. For example, we saw verbal comments where people clarified they had chosen the angry emoticon response not because they were angry at the person who posted, but rather at the message in the item being shared. Similarly, the wow emoticon response is vague with regards to whether it offers a positive or negative sentiment.

In terms of flow, the tools all freely facilitate sharing of knowledge objects. Platforms that heavily focused on verbal message exchange and sharing (e.g., Facebook, Twitter) more robustly support flow in. Tools where creation occurs or creations are hosted (e.g., YouTube), more robustly support flow out.

Through our analysis, we have seen how challenging it is to definitively identify different NKA through analysis of social media archives. The challenge is not in finding indicators, but in knowing that those indicators are always present to mark a networked knowledge activity and reliably indicate that activity. Although this may seem to be a disheartening finding, it is not surprising and does not render NKA useless as an analytic framework. However, it once again affirms what has been learned via many studies in the field of learning analytics: Evidence of user actions in a data archive cannot be accurately interpreted for user intent.

This work is not yet complete. Next steps include surveys, which will be used to confirm findings from this initial analysis and ascertain frequency of the NKAs from the user perspective. Through this continued work, we can better learn how to identify learning indicators on social networking sites and offer both meaningful ways of assessing whether knowledge activities have occurred from data archives. 


\section{REFERENCES}

Aaen, J. and C. Dalsgaard (2016). "Student Facebook groups as a third space: Between social life and schoolwork." Learning, Media and Technology 41(1): 160-186.

Adalberon, E. and R. Säljö (2017). "Informal use of social media in higher education: A case study of Facebook groups." Nordic Journal of Digital Literacy 12(04): 114-128.

Al-Heeti, A. (2019) "For Facebook and Instagram, axing likes may not be about mental health after all." cnet news.

Anderson, M. and J. Jiang (2018). Teens, social media \& technology 2018. Washington, D.C., Pew Research.

Anderson, M. and A. Perrin (2017). Tech adoption climbs among older adults. Washington, D.C., Pew Research Center.

Bagdy, L. M., V. P. Dennen, S. A. Rutledge, J. T. Rowlett and S. Burnick (2018). Teens and social media: A case study of high school students' informal learning practices and trajectories. Proceedings of the 9th International Conference on Social Media and Society. New York, NY, ACM: 241-245.

Bloom, B. S. (1956). Taxonomy of educational objectives. Vol. 1: Cognitive domain. New York, NY, McKay.

Bond, R. and S. Messing (2015). "Quantifying social media's political space: Estimating ideology from publicly revealed preferences on Facebook." American Political Science Review 109(1): 62-78.

Bridgstock, R. (2019). Employability and career development learning through social media: Exploring the potential of LinkedIn. Challenging future practice possibilities, Brill Sense: 143-152.

Brown Jarreau, P., N. S. Dahmen and E. Jones (2019). "Instagram and the science museum: A missed opportunity for public engagement." Journal of Science Communication 18(2): A06.

Carpenter, J. P. and D. G. Krutka (2015). "Engagement through microblogging: Educator professional development via Twitter." Professional Development in Education 41(4): 707-728.

Chambers, D. (2013). Social media and personal relationships: Online intimacies and networked friendship. London, Palgrave Macmillan.

Choi, G. Y. and E. Behm-Morawitz (2017). "Giving a new makeover to STEAM: Establishing YouTube beauty gurus as digital literacy educators through messages and effects on viewers." Computers in Human Behavior 73: 80-91.

Dennen, V. P. (2019). Activity interactions in online courses. Handbook of Distance Education. M. G. Moore and W. Diehl. New York, NY, Routledge.

Fish, L. (2018). Exploring the motivations underlying the use of paralinguistic digital affordances on Facebook, University of Tasmania.

Gagne, R. M. (1984). "Learning outcomes and their effects: Useful categories of human performance." American Psychologist 39(4): 377-385.

Greenhalgh, S. P. and M. J. Koehler (2017). "28 Days Later: Twitter Hashtags as "Just in Time" Teacher Professional Development." TechTrends 61(3): 273-281.

Greenhow, C. and C. Lewin (2016). "Social media and education: Reconceptualizing the boundaries of formal and informal learning." Learning, Media and Technology 41(1): 6-30.

Hayes, R. A., C. T. Carr and D. Y. Wohn (2016). "One click, many meanings: Interpreting paralinguistic digital affordances in social media." Journal of Broadcasting \& Electronic Media 60(1): 171-187.

Hu, S., K. T. Torphy, A. Opperman, K. Jansen and Y.-J. Lo (2018). "What do teachers share within socialized knowledge communities: A case of Pinterest." Journal of Professional Capital Community.

Krathwohl, D. R. and L. W. Anderson (2009). A taxonomy for learning, teaching, and assessing: A revision of Bloom's taxonomy of educational objectives. New York, NY, Longman.

Lapolla, K. (2014). "The Pinterest project: Using social media in an undergraduate second year fashion design course at a United States University." Art, Design \& Communication in Higher Education 13(2): 175-187.

Meishar-Tal, H., G. Kurtz and E. Pieterse (2012). "Facebook groups as LMS: A case study." The International Review of Research in Open \& Distributed Learning 13(4): 33-48.

Milligan, C., A. Littlejohn and A. Margaryan (2014). "Workplace learning in informal networks." Journal of Interactive Media in Education 1(6).

Moghavvemi, S., A. Sulaiman, N. I. Jaafar and N. Kasem (2018). "Social media as a complementary learning tool for teaching and learning: The case of YouTube." The International Journal of Management Education 16(1): 37-42.

Perrin, A. and M. Anderson (2019). Share of U.S. adults using social media, including Facebook, is mostly unchanged since 2018. Washington, D.C., Pew Research Center.

Salomon, D. (2013). "Moving on from Facebook: Using Instagram to connect with undergraduates and engage in teaching and learning." College \& Research Libraries News 74(8): 408-412.

Wang, Q., H. L. Woo, C. L. Quek, Y. Yang and M. Liu (2012). "Using the Facebook group as a learning management system: An exploratory study." British Journal of Educational Technology 43(3): 428-438.

Wohn, D. Y., C. T. Carr and R. A. Hayes (2016). "How Affective Is a "Like"?: The Effect of Paralinguistic Digital Affordances on Perceived Social Support." Cyberpsychology, Behavior, and Social Networking 19(9): 562-566. 